\section{Isolated tubal torsion: A rare cause of acute abdomen in childhood}

\author{
Berat Dilek Demirel, Sertac Hancioglu, \\ Unal Bicakci, Ender Ariturk, \\ Ferit Bernay \\ Department of Pediatric Surgery, \\ Ondokuz Mayis University, Samsun, \\ Turkey
}

\begin{abstract}
Isolated fallopian tube torsion is a rare cause of acute abdomen mostly seen in women of reproductive age. It is often diagnosed during surgery. In this study we aimed to present a case of isolated fallopian tube torsion in a child. An 11-year-old girl presented with abdominal pain. With the help of radiological evaluation we diagnosed. It should be kept in mind that even if ovarian blood flow is normal in girls with abdominal pain, it may be an isolated tubular torsion and requires immediate intervention.
\end{abstract}

\section{Introduction}

Isolated tubal torsion (ITT) is a rare cause of acute abdomen, which is commonly seen during reproductive age and may result in loss of fertility. ${ }^{1}$ Torsion of the ovary and salpinx in which the ovary and fallopian tube rotate around the broad ligament is more commonly observed; however, isolated ovarian torsion in which only the ovary rotates around the mesovarium or ITT in which only the fallopian tube rotates around the mesosalpinx is more rarely observed. ${ }^{2}$ ITT is observed in 1 in 1.5 million women of reproductive age. ${ }^{1,2}$ Although it is less common in children, there is no information regarding its prevalence rate.

The most common complaint is abdominal pain with sudden onset. Vomiting, fever, nausea and urinary symptoms may be accompanied. A classic acute abdomen may be found and is usually diagnosed by performing diagnostic surgery. ${ }^{3-5}$ In this case report, we present a case of ITT that was diagnosed preoperatively and identified during childhood. It was evaluated with the help of literature.

\section{Case Report}

An 11-year-old female who had not yet started to menstruate presented with gradually increasing abdominal pain for 2 days. The pain was primarily in the right lower quadrant of the abdomen and in the form of a cramp. The patient did not suffer from nausea, vomiting or fever-like complaints. Physical examination revealed diffuse tenderness on all lower quadrants, mainly in the right lower quadrant. Hemogram, biochemical parameters, $C$ reactive protein value and urinary values were found to be normal in the laboratory analysis. Her vital findings were stable. Standing direct abdominal x-ray revealed no pathological findings. Transabdominal Doppler ultrasonographic examination revealed normal blood circulation in both ovaries. However, there was an anechoic mass unrelated to the ovaries and uterus in the pelvis. Magnetic resonance imaging revealed a paraovarian, cystic lesion of $5 \times 4 \mathrm{~cm}$ size on the posterior side of the bladder which may tubal origin, an increase in the size of the right ovary, an edematous appearance on the stroma, and rotation of tubal pedicle between the cyst and the right ovary, involving the vascular structures (Figure 1). The patient underwent laparoscopy with the pre-diagnosis of right tubal torsion. The uterus and the circulation in both the ovaries were found to be normal during laparoscopic exploration. The right ovary was odematous and had increased in size (Figure 2). Tubal torsion was observed in the pelvis. The tube had rotated four times around its axis and was detorsioned. The salpinx comprised necrotic tissue of $5 \times 4 \mathrm{~cm}$ diameter and edematous tissue of 5-cm length (Figure 3). The patient underwent right salpingectomy. The surgery was laparoscopically performed. Microscopic evaluation revealed necrotic and hemorrhagic areas of infiltration with polymorphonuclear leucocytes. Histopathological evaluation revealed tubal tissue with diffuse necrosis. She recovered without any postoperative complications.

\section{Discussion}

ITT is a very rare condition among gynecological urgent conditions. It is more common in the reproductive age, but is very rare in the prepubertal and perimenopausal age. 4 This patient was 11-years old and had not started to menstruate. It should be considered that ovarian and paratubal masses, pregnancy and trauma may also play a role in the etiology of ITT as well as tubal causes, such as congenital anomalies of the tuba, a long mesosalpinx, hydrosalpinx, hematosalpinx, tubal ligation or tubal surgical interventions. ${ }^{5-9} \mathrm{We}$ could not identify a known etiology in this case. However, a longer tube was observed in our patient. The tuba was edematous and necrotic; hence, correct length assessment was not possible.

Because ITT does not have a specific
Correspondence: Berat Dilek Demirel, Department of Pediatric Surgery, Ondokuz Mayis University, Körfez Mahallesi, 19 Mayis Ünv. Kurupelit Kampüsü, 55105 Atakum/Samsun, Turkey.

E-mail: bdayhan@hotmail.com

Key words: Acute abdomen, isolated tubal torsion, prepubertal, torsion.

Contributions: the authors contributed equally.

Funding: none.

Conflict of interest: the authors declare no potential conflict of interest.

Received for publication: 23 January 2018.

Revision received: 3 March 2018.

Accepted for publication: 5 March 2018.

This work is licensed under a Creative Commons Attribution NonCommercial 4.0 License (CC BY-NC 4.0).

CCopyright B.D. Demirel et al. 2018

Licensee PAGEPress, Italy

Pediatric Reports 2018; 10:7604

doi:10.4081/pr.2018.7604

clinical complaint, laboratory finding and radiologically specific finding, it is commonly diagnosed by performing laparoscopy or laparatomy. ${ }^{6}$ However, in recent years, the number of cases diagnosed preoperatively using Doppler ultrasonography, computed tomography or magnetic resonance imaging is increasing. ${ }^{7}$ ITT was considered after using transabdominal Doppler ultrasonography in our patient. The preoperative diagnosis was strengthened by magnetic resonance imaging. Since delay in the diagnosis causes irreversible tubal injury, it may result in loss of fertility. Therefore, early diagnosis and treatment are essential. ${ }^{8}$ Narayanan et al. reported their preoperative radiological evaluation in their study with 10 pediatric patients who underwent surgery because of ITT. They preoperatively diagnosed 9 patients (five by ultrasound, four by tomography). They considered ultrasound to be the first choice for diagnosis of every patient with pelvic pain. ${ }^{9}$

Due to its rare occurrence, very few studies have been published. When the reported cases are evaluated, tubal torsion was more common on the right side, as observed in our case. ${ }^{10}$ This may be attributed to anatomical reasons. Patients who cannot be diagnosed preoperatively generally undergo surgery with the pre-diagnosis of acute appendicitis because it is more commonly observed on the right side. With the help of minimally invasive surgical methods, even if ITT is not preoperatively diagnosed, the required surgical procedure can be performed by observing the tubal torsion when diagnostic laparoscopy is 
performed considering acute abdomen. However, it is more important to make a diagnosis in early-stage patients who do not clinically develop the disease. Tubal circulation can be restored and the development of necrosis can be prevented in patients at this stage of the disease. Therefore, women presenting with abdominal pain should be radiologically evaluated regardless of the age group. ITT can be diagnosed by performing transabdominal ultrasonography. In earlystage cases, the tuba is detorsioned by surgi-

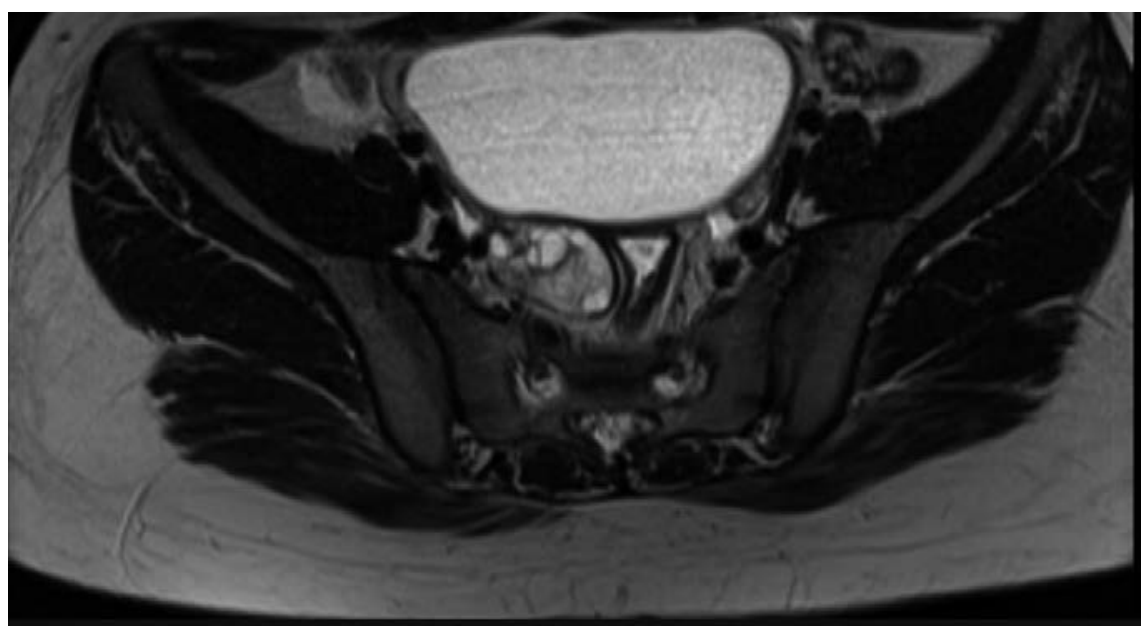

Figure 1. Magnetic resonance imaging of isolated tubal torsion.

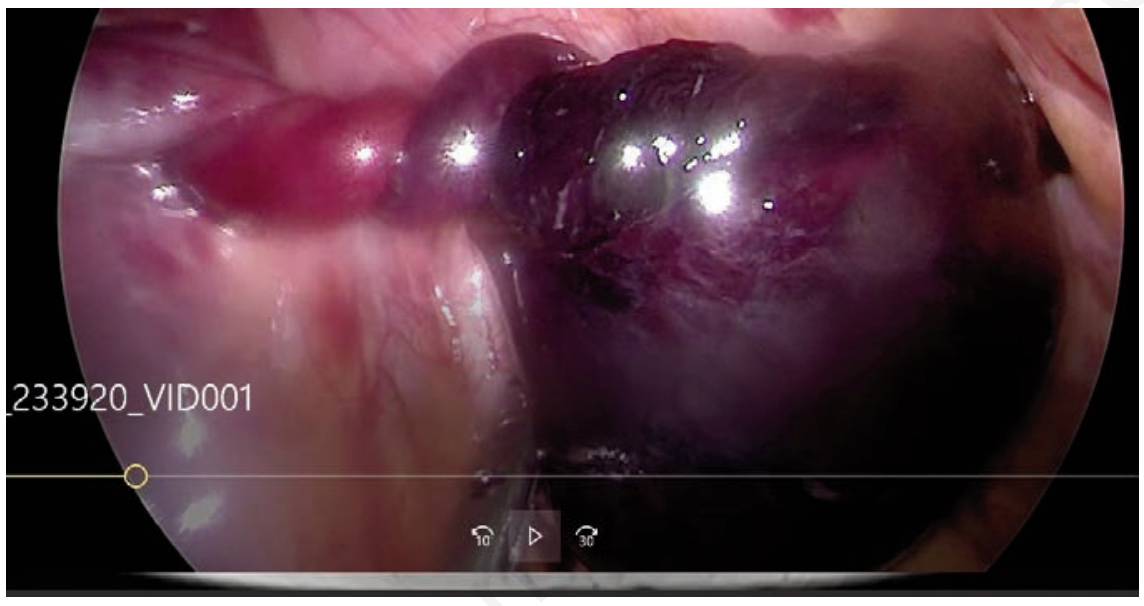

Figure 2. Laparoscopic exploration of pelvic organs.

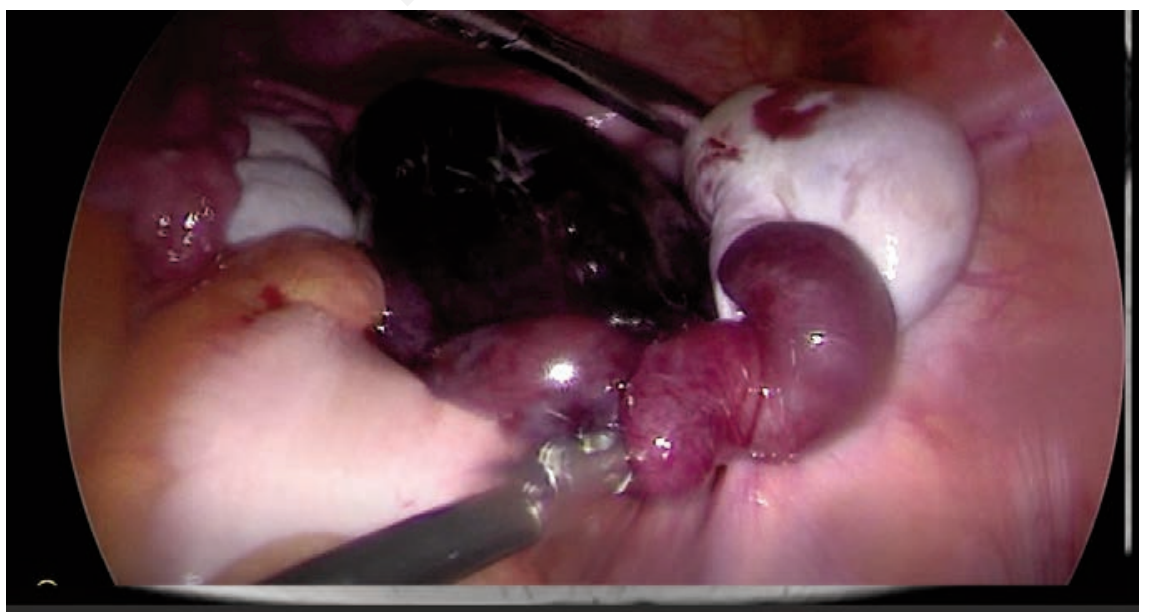

Figure 3. Tubal torsion. cal treatment; however, salpingectomy is necessary in cases where the tuba is necrotic, as in our case. Diagnostic laparoscopy should be performed in cases where a definitive diagnosis cannot be made clinically and radiologically.

\section{Conclusions}

Isolated tubal torsion should absolutely be considered in girls presenting with an acute abdomen.

\section{References}

1. Gaied F, Emil S, Lo A, et al. Laparoscopic treatment of isolated salpingeal torsion in children: case series and a 20-year review of the literature. J Laparoendosc Adv Surg Tech A 2012;22:941-7.

2. Merlini L, Anooshiravani M, Vunda A, et al. Noninflammatory fallopian tube pathology in children. Pediatr Radiol 2008;38:1330-7 .

3. Comerci G, Colombo FM, Stefanetti M, Grazia $\mathrm{G}$. Isolated fallopian tube torsion: a rare but important event for women of reproductive age. Fertil Steril 200890:1198.e23-5.

4. Ding DC, Hsu S, Kao SP. Isolated torsion of the hydrosalpinx in a postmenopausal woman. JSLS 2007; 11:252-4.

5. Webster KW, Scott SM, Huguelet PS. Clinical predictors of isolated tubal torsion: a case series. J Pediatr Adolesc Gynecol 2017;30:578-81.

6. Macedo M, Kim B, Khoury R, Narkiewicz L. A rare case of right lower quadrant abdominal pain. Am J Emerg Med 2017;25:668.

7. Fadıloglu E, Dur R, Demirdag E, et al. Isolated tubal torsion: Successful preoperative diagnosis of five cases using ultrasound and management with laparoscopy. Turk J Obstet Gynecol 2017;14:187-90.

8. Schuh AM, Klein EJ, Allred RJ, et al. Pediatric adnexal torsion: not just a postmenarchal problem. J Emerge Med 2017;52:169-75.

9. Narayanan S, Bandarkar A, Bulas DI. Fallopian tube torsion in the pediatric age group: radiologic evaluation. J Ultrasound Med 2011;51:244-7.

10. Lo LM, Chang SD, Lee CL, Liang CC. Clinical manifestations in women with isolated fallopian tubal torsion; a rare but importanat entitiy. Aust NZ J Obstet Gynaecol 2011;51:244-7. 